\title{
Comparação dos Resultados do Tratamento Cirúrgico da Incontinência Urinária de Esforço por Três Diferentes Técnicas Cirúrgicas
}

\author{
Comparison of the Results of the Treatment of Stress Urinary Incontinence with Three \\ Different Surgical Procedures
}

José Geraldo Lopes Ramos, Nilton Leite Xavier, Andrea Prestes Nácul

Ângela Erguy Zucatto, Eduardo Lange Hentschel

\begin{abstract}
RESUMO
Objetivo: analisar a prevalência de recidivas de incontinência urinária de esforço (IUE) tratada com diferentes técnicas cirúrgicas após pelo menos 2 anos de seguimento.

Pacientes e Métodos: avaliamos 55 pacientes com diagnóstico de IUE que submeteram-se à cirurgia para sua correção no Serviço de Ginecologia do Hospital de Clinicas de Porto Alegre, no periodo de 1992 a 1996. O seguimento pós-cirúrgico nesse Serviço foi superior a 2 anos. As pacientes foram divididas em 3 grupos conforme a técnica cirúrgica empregada: Kelly-Kennedy ( $n=24)$, Burch $(n=23)$ e Marshall-Marchetti-Krantz $(n=8)$.

Resultados: não foram encontradas diferenças significativas quanto ao tempo de recidiva, idade na época da cirurgia e da recidiva, estado menopausal, uso de terapia de reposição hormonal (TRH), número de gestações e antecedentes de parto via vaginal. $O$ número de casos com perineoplastia posterior foi maior no grupo de cirurgia de Kelly-Kennedy, sem, contudo, influir na recidiva. O grupo de cirurgia de Burch apresentou um tempo de menopausa maior quando da cirurgia $(p<0,05)$.

Conclusão: a taxa de recidiva com emprego das técnicas de Kelly-Kennedy, Burch e MarshallMarchetti-Krantz foi respectivamente 29,2, 39,1 e 50\%, não diferindo do ponto de vista estatístico. A pesquisa de modificadores de risco para a incontinência urinária genuína não foi estatisticamente diferente nos três grupos estudados. Observou-se, contudo, que a totalidade das pacientes com cirurgia prévia recidivaram.
\end{abstract}

PALAVRAS-CHAVE: Incontinência urinária de esforço. Cirurgia vaginal. Terapia de reposição hormonal.

\section{Introdução}

Com o aumento da expectativa de vida das mulheres, a incontinência urinária tornou-se uma causa importante de limitação das atividades nos grupos etários mais avançados. Cinqüenta e sete por cento das mulheres entre 45 e 64 anos apresentam a disfunção ${ }^{1}$, sendo que a meta-

Departamento de Ginecologia e Obstetrícia da Faculdade de Medicina da Universidade Federal do Rio Grande do Sul

Correspondência:

José Geraldo Lopes Ramos

R. Ramiro Barcelos 2350 s. 1127

90035-007 - Porto Alegre - RS

FAX: (51) 333-1585 de dos casos estão associados a prolapsos uretrovesicais, para os quais o tratamento primário é cirúrgico ${ }^{2}$. Assim, parece evidente o interesse em se buscar um melhor conhecimento a respeito das técnicas cirúrgicas empregadas na correção da incontinência urinária de esforço (IUE).

As avaliações clínicas mostram taxas de recorrência que variam de 5 a $20 \%$ nas abordagens por via abdominal e $10-64 \%$ quando se utiliza a via vaginal ${ }^{3}$. Enzelsberger et al. ${ }^{1}$ descreveram $89 \%$ de cura da IUE em 32-48 meses de seguimento após o procedimento de Burch. Girão et al. ${ }^{4}$ encontraram uma taxa de sucesso de $84,5 \%$ com o emprego da via vaginal e $82,1 \%$ na via abdominal após seguimento de 1 a 194 meses. Já 
Marana et al. ${ }^{5}$, reavaliando as pacientes após 54 a 66 meses, observaram uma prevalência de recorrência de 78,9\% com a técnica de KellyKennedy (KK) e de $40 \%$ quando empregada a técnica de Burch. Colombo et al. ${ }^{6}$ encontraram, após seguimento de 1-3 anos, uma taxa de cura igual a $100 \%$ na técnica de Burch e $72 \%$ quando utilizada a técnica de Kelly-Kennedy.

No estudo de Beck et al. ${ }^{7}$, foi avaliado o uso da técnica de Kelly-Kennedy original e modificada para correção de IUE por um período de até 25 anos. O índice de cura foi de $75 \%$ na técnica original e $91 \%$ na técnica modificada. Pacientes que haviam realizado cirurgias prévias para incontinência urinária tiveram seu indice de cura significativamente reduzido.

Diversos fatores e variáveis têm sido descritos como associados a recidivas de IUE após correção cirúrgica, entre eles: cirurgia pélvica prévia, realização de perineoplastia posterior associada, idade, história obstétrica, obesidade, constipação e tosse crônicas, hipoestrogenismo, habilidade e familiaridade do cirurgião com a técnica cirúrgica utilizada ${ }^{4,7,8,9}$. Outros fatores, de alguma forma, estão relacionados a alterações anatômicas do assoalho pélvico, perda do ângulo uretro-vesical e/ou a um aumento da pressão intra-abdominal ${ }^{3,8}$.

O presente estudo foi delineado com o objetivo de avaliar a prevalência de recidivas e estimar a média de duração da efetividade da correção cirúrgica nas diferentes técnicas empregadas.

\section{Pacientes e Métodos}

Foram avaliadas retrospectivamente 55 pacientes com diagnóstico de IUE submetidas à cirurgia para correção no Serviço de Ginecologia e Obstetrícia do Hospital de Clínicas de Porto Alegre (HCPA) no período de 1992 a 1996 e que mantiveram seguimento nesse Serviço por pelo menos 2 anos.

O diagnóstico de IUE baseou-se na história de perda urinária aos esforços referida pelas pacientes associada à presença de defeitos anatômicos. Todas as pacientes apresentavam defeitos anatômicos que justificavam a incontinência urinária.

As pacientes foram divididas em três grupos, segundo o tipo de cirurgia realizada: grupo 1 , cirurgia de Kelly-Kennedy (KK); grupo 2, cirurgia de Burch e grupo 3, cirurgia de MarshallMarchetti-Krantz (MMK). Os grupos foram comparados em relação à idade na época da cirurgia, número de gestações e de partos via baixa, tempo de seguimento pós-cirúrgico, recidiva da incontinência urinária, realização de cirurgia prévia para correção de incontinência urinária genuína, perineoplastia posterior associada, estado menopausal por ocasião da cirurgia, uso de terapia de reposição hormonal (TRH) e anos de menopausa na recidiva. Posteriormente, as mesmas variáveis foram analisadas dentro de cada grupo em relação à presença ou não de recidiva, assim como à época em que a recidiva ocorreu nos diferentes grupos.

O estudo urodinâmico foi realizado em todas as pacientes com cirurgia prévia, já que todas as pacientes apresentavam indicação de correção cirúrgica pela alteração anatômica. A indicação de cirurgia por via vaginal baseou-se na presença de prolapso proeminente de paredes vaginais ou mesmo de prolapso uterino.

Para análise estatística foram utilizados os testes do $\chi^{2}$, de Fisher e análise de variância. Os dados são apresentados como média \pm desvio padrão. O nivel de significância foi estabelecido em 0,05. O projeto foi avaliado e aprovado pelo Comitê de Pesquisa e Ética do HCPA.

\section{Resultados}

Das 55 pacientes que fizeram parte do estudo, 24 (43,6\%) submeteram-se à abordagem por via vaginal, ao passo que em 31 pacientes a correção foi realizada por via abdominal, sendo que em 23 $(41,8 \%)$ a técnica utilizada foi a de Burch e em 8 $(14,6 \%)$ foi a de Marshall-Marchetti-Krantz.

No grupo em que a técnica de Kelly-Kennedy foi utilizada, com exceção de 1 caso, as pacientes foram também submetidas a colpoperineoplastia posterior (CPPP). Já no grupo da cirurgia de Burch, aproximadamente metade das pacientes o fizeram, ao passo que no MMK apenas 1 caso associou-se a CPPP. Em face do fato de o grupo MMK apresentar um pequeno número de pacientes foi utilizado o teste exato de Fisher pareando os grupos dois a dois. O grupo de MMK teve uma prevalência significativamente maior de cirurgia prévia que o grupo de KK ( $p=0,0393)$. Os grupos não diferiram significativamente quanto aos demais modificadores de risco analisados. Os dados estão apresentados na Tabela 1.

A prevalência de recidiva da incontinência urinária de esforço nas pacientes tratadas com cirurgia de Kelly-Kennedy foi de 29,2\%. Na técnica de Burch, 39,1\% das pacientes recidivaram, ao passo que na de Marshall-Marchetti-Krantz a taxa atingiu $50,0 \%$. As três técnicas não diferiram es- 
tatisticamente quanto à prevalência de recidiva de incontinência urinária de esforço $(\mathrm{p}=0,53)$. No grupo abordado pela técnica de MarshallMarchetti-Krantz, 37,5\% (3) das pacientes apresentavam história de cirurgia prévia. Ao excluir- mos as pacientes com cirurgia prévia, a prevalência de recidiva nos grupos 1,2 e 3 foi respectivamente de $26,1,22,2$ e $20,0 \%(p=0,93)(T a-$ bela 2).

Tabela 1 - Características das pacientes tratadas com três diferentes técnicas cirúrgicas para correção de incontinência urinária de esforço.

\begin{tabular}{|c|c|c|c|c|}
\hline $\begin{array}{l}\text { Características da amostra na } \\
\text { época da cirurgia }\end{array}$ & $\begin{array}{l}\text { Kelly-Kennedy } \\
n=24\end{array}$ & $\begin{array}{l}\text { Burch } \\
n=23\end{array}$ & $\begin{array}{c}\text { Marshall- } \\
\text { Marchetti-Krantz } \\
n=8\end{array}$ & $\mathrm{p}$ \\
\hline Gestações (média — DP) & $4,6( \pm 2,6)$ & $3,8( \pm 2,6)$ & $4,6 \pm 3,2$ & NS \\
\hline Partos via vaginal (média $\pm \mathrm{DP}$ ) & $3,5( \pm 2,2)$ & $3,2( \pm 2,4)$ & $3,7 \pm 3,3$ & NS \\
\hline Uso de TRH (n) & 6 & 6 & 2 & NS \\
\hline Cirurgia prévia (n) & 1 & 5 & 3 & 0,05 \\
\hline Perineoplastia posterior associada (n) & 23 & 11 & 1 & 0,00001 \\
\hline
\end{tabular}

TRH: Terapia de reposição hormonal.

Tabela 2 - Prevalência de recidiva de incontinência urinária com uso de diferentes técnicas cirúrgicas de acordo com a presença ou não de cirurgia prévia.

\begin{tabular}{llll}
\hline Recidiva & Kelly-Kennedy & Burch & Marshall-Marchetti-Krantz \\
\hline Sem cirurgia prévia n (\%) & $6 / 23(26,1)$ & $4 / 18(22,2)$ & $1 / 5(20)$ \\
Com cirurgia prévia n $(\%)$ & $1 / 1(100)$ & $5 / 5(100)$ & $3 / 3(100)$ \\
Grupo total & $7(29,2)$ & $9(39,1)$ & $4(50)$ \\
\hline
\end{tabular}

A média de meses que decorreram do procedimento cirúrgico até a recidiva da incontinência urinária foi de $21,6 \pm 15,3$ meses, $19,9 \pm 20,1$ meses e 34,0 $\pm 32,9$ meses, respectivamente nos grupos 1,2 e $3(p=0,54)$.

A fim de evitar fatores de confusão que comprometessem os resultados encontrados, as pacientes que recidivaram foram comparadas com as que não apresentaram recidiva em cada um dos grupos isoladamente quanto à idade, número de gestações e de partos via vaginal, estado menopausal, anos de menopausa, utilização de terapia de reposição hormonal, realização de cirurgia prévia para o mesmo fim e perineoplastia posterior associada. As variantes acima não influenciaram a taxa de recidiva de incontinência urinária dentro de cada grupo (Tabela 3).

Em análise subseqüente, comparamos as pacientes que tiveram ou não recidiva entre os três diferentes grupos no que diz respeito aos seguintes fatores: tempo de recidiva, idade por ocasião da cirurgia e da recidiva, cirurgia prévia, freqüência de pós-menopáusicas na recidiva, anos de menopausa, estado menopausal na época da cirurgia, terapia de reposição hormonal, realização de perineoplastia posterior e número de gestações e de partos via vaginal.

Comparando as pacientes que recidivaram quanto às diferentes técnicas cirúrgicas empregadas, o número de anos após a menopausa por ocasião da cirurgia foi significativamente maior no grupo Burch e Kelly-Kennedy do que no grupo Marshall-Marchetti-Krantz $(\mathrm{p}<0,05)$. Comparando as que não recidivaram entre as diferentes abordagens, só houve diferença estatisticamente significativa quanto à realização de perineoplastia posterior associada ou não. O grupo submetido à cirurgia de Kelly-Kennedy teve número maior de perineoplastias posteriores associadas do que os outros dois $(\mathrm{p}<0,05)$. Os dados estão demonstrados na Tabela 4. 
Tabela 3 - Comparação dos modificadores de risco em relação à recidiva da incontinência urinária nos grupos submetidos às diferentes técnicas cirúrgicas

\begin{tabular}{|c|c|c|c|c|c|c|c|c|c|}
\hline \multirow[b]{2}{*}{ Modificadores de risco } & \multicolumn{3}{|c|}{ Kelly-Kennedy } & \multicolumn{3}{|c|}{ Burch } & \multicolumn{3}{|c|}{ Marshall-Marchetti-Krantz } \\
\hline & $\begin{array}{l}\text { Com recidiva } \\
\qquad(n=7)\end{array}$ & $\begin{array}{l}\text { Sem recidiva } \\
\qquad(n=17)\end{array}$ & $p$ & $\begin{array}{l}\text { Com recidiva } \\
\qquad(\mathrm{n}=9)\end{array}$ & $\begin{array}{l}\text { Sem recidiva } \\
\qquad(n=14)\end{array}$ & $\mathrm{p}$ & $\begin{array}{l}\text { Com recidiva } \\
\qquad(n=4)\end{array}$ & $\begin{array}{l}\text { Sem recidiva } \\
\qquad(n=4)\end{array}$ & $\mathbf{P}$ \\
\hline $\begin{array}{l}\text { Idade } \\
\text { (média } \pm \text { DP) }\end{array}$ & $50,1 \pm 10,9$ & $47,5 \pm 9,9$ & NS & $45,6 \pm 10,6$ & $47,7 \pm 8,8$ & NS & $44,2 \pm 10,4$ & $50,0 \pm 5,6$ & NS \\
\hline $\begin{array}{l}\text { Gestações } \\
\text { (média } \pm \text { DP) }\end{array}$ & $4,1 \pm 3,2$ & $4,8 \pm 2,3$ & NS & $4,4 \pm 2,9$ & $3,4 \pm 2,4$ & NS & $4,5 \pm 3,8$ & $4,7 \pm 2,9$ & NS \\
\hline $\begin{array}{l}\text { Partos via vaginal } \\
\text { (média } \pm \mathrm{DP} \text { ) }\end{array}$ & $3,2 \pm 2,9$ & $3,7 \pm 1,9$ & NS & $3,7 \pm 3,0$ & $2,8 \pm 2,1$ & NS & $3,7 \pm 4,1$ & $3,7 \pm 2,8$ & NS \\
\hline $\begin{array}{l}\text { Pós-menopáusicas } \\
\text { na cirurgia (n) }\end{array}$ & 3 & 6 & NS & 3 & 5 & NS & 2 & 2 & NS \\
\hline $\begin{array}{l}\text { Anos de menopausa } \\
\text { na cirurgia (média } \pm D P \text { ) }\end{array}$ & $10,0 \pm 3,0$ & $7,1 \pm 8,4$ & NS & $17,3 \pm 10,7$ & $9,6 \pm 11,3$ & NS & $2,5 \pm 0,7$ & $14,0 \pm 11,3$ & NS \\
\hline Uso de TRH (n) & 3 & 3 & NS & 4 & 2 & NS & 1 & 1 & NS \\
\hline Cirurgia prévia (n) & 1 & 0 & NS & 2 & 3 & NS & 3 & 0 & NS \\
\hline $\begin{array}{l}\text { Perineoplastia posterior } \\
\text { associada (n) }\end{array}$ & 6 & 17 & NS & 4 & 7 & NS & 1 & 0 & NS \\
\hline
\end{tabular}

TRH: Terapia de reposição hormonal.

Tabela 4 - Comparação dos modificadores de risco em pacientes que recidivaram entre as três técnicas cirúrgicas empregadas.

\begin{tabular}{|c|c|c|c|c|c|c|c|c|}
\hline \multirow[t]{2}{*}{ Modificadores de risco } & \multicolumn{2}{|c|}{$\begin{array}{c}\text { Kelly-Kennedy } \\
\text { incontinente continente }\end{array}$} & \multicolumn{2}{|c|}{$\begin{array}{c}\text { Burch } \\
\text { incontinente continente }\end{array}$} & \multicolumn{2}{|c|}{$\begin{array}{l}\text { Marshall-Marchett-Krantz } \\
\text { incontinente continente }\end{array}$} & \multicolumn{2}{|c|}{$p$} \\
\hline & $(n=7)$ & $(n=7)$ & $(n=9)$ & $(n=14)$ & $(n=4)$ & $(n=4)$ & & \\
\hline $\begin{array}{l}\text { Tempo pós-cirúrgico sem } \\
\text { recorrência (meses) }\end{array}$ & $21,5+15,2$ & - & $19,8 \pm 20,1$ & & $34,0 \pm 32,8$ & - & NS & - \\
\hline Idade (média $\pm \mathrm{DP}$ ) & $50,1 \pm 10,9$ & $47,5 \pm 9,9$ & $45,6 \pm 10,6$ & $47,7 \pm 8,8$ & $44,2 \pm 10,4$ & $50,0 \pm 5,6$ & NS & NS \\
\hline Gestações (média \pm DP) & $4,1 \pm 3,2$ & $4,8 \pm 2,3$ & $4,4 \pm 2,9$ & $3,4 \pm 2,4$ & $4,5 \pm 3,8$ & $4,7 \pm 2,9$ & NS & NS \\
\hline $\begin{array}{l}\text { Partos via vaginal } \\
\text { (média } \pm \text { DP) }\end{array}$ & $3,2 \pm 2,9$ & $3,7 \pm 1,9$ & $3,7 \pm 3,0$ & $2,8 \pm 2,1$ & $3,7 \pm 4,1$ & $3,7 \pm 2,8$ & NS & NS \\
\hline Idade na recidiva (anos) & $52,0 \pm 11,5$ & - & $47,3 \pm 11,1$ & - & $48,0 \pm 9,4$ & - & NS & - \\
\hline $\begin{array}{l}\text { Pós-menopausa } \\
\text { na cirurgia (n) }\end{array}$ & 3 & 6 & 3 & 5 & 2 & 2 & NS & NS \\
\hline $\begin{array}{l}\text { Anos de menopausa } \\
\text { na cirurgia (média } \pm \text { DP) }\end{array}$ & $10,0 \pm 3,0$ & $7,1 \pm 8,4$ & $17,3 \pm 10,7$ & $9,6 \pm 11,3$ & $2,5 \pm 0,7$ & $14,0 \pm 11,3$ & 0,04 & NS \\
\hline $\begin{array}{l}\text { Anos de menopausa } \\
\text { na recidiva }\end{array}$ & $9,5 \pm 6,2$ & - & $14,5 \pm 13,7$ & - & $4,0 \pm 3,6$ & - & NS & - \\
\hline Uso de TRH (n) & 3 & 3 & 4 & 2 & 1 & 1 & NS & NS \\
\hline Cirurgia prévia (n) & 1 & 0 & 2 & 3 & 3 & 0 & NS & - \\
\hline $\begin{array}{l}\text { Perineoplastia posterior } \\
\text { associada (n) }\end{array}$ & 6 & 17 & 4 & 7 & 1 & 0 & NS & 0,00008 \\
\hline
\end{tabular}

TRH: Terapia da reposição hormonal. 


\section{Discussão}

A incontinência urinária de esforço, também chamada de verdadeira, de estresse ou genuína, é definida como um sinal, sintoma ou condição de perda involuntária de urina quando a pressão intravesical ultrapassa a pressão uretral máxima na ausência de contração do detrusor e representa problema social ou higiênico ${ }^{4,8}$. Alterações anatômicas decorrentes da modificação do posicionamento vesical, com o colo situado em posição mais baixa, fazem com que, na incontinência urinária de esforço, a distribuição da pressão uretral se altere e ocorra diminuição de sua resistência, com perda urinária ${ }^{3}$. Em cerca de $30 \%$ dos casos, há associação de urgência com incontinência urinária de esforço ${ }^{3}$, sendo a primeira, freqüentemente, a responsável pelas re$\operatorname{cidivas}^{10}$.

A análise de séries de casos incorre em uma seqüência de dificuldades e fatores de confusão, principalmente quando o assunto abordado refere-se à incontinência urinária feminina. O número excessivo de tipos de técnicas cirúrgicas e os múltiplos fatores de risco associados fazem com que uma análise objetiva dos sucessos terapêuticos seja dificultada.

Um ponto importante a ser sempre analisado é quanto ao tempo de observação após o procedimento cirúrgico. As taxas de falhas dos procedimentos cirúrgicos devem sempre ser analisadas após pelo menos dois anos de observação, conforme a nossa metodologia, ressaltando-se que as cifras de recorrência continuam crescendo após este periodo. Baden et al. ${ }^{11}$ encontraram uma diminuição na eficácia dos procedimentos cirúrgicos de $84,1 \%$ para $75 \%$ quando comparados dois e sete anos de controle. Parece que a estabilização da taxa de cura ocorre após cerca de cinco a sete anos de observação $0^{5,11}$.

O uso de técnicas de investigação para causas não-anatômicas para incontinência urinária também é outro fator que pode diferenciar taxas de recidiva. No nosso Serviço temos preconizado a realização de testes urodinâmicos em todas as pacientes com outros sintomas urinários concomitantes, que não apresentem defeitos anatômicos que justifiquem a incontinência ou que apresentem história de cirurgia prévia para incontinência urinária.

No presente estudo, embora não-significativo do ponto de vista estatístico, a técnica de MMK se mostrou superior à de Burch que, por sua vez, foi mais efetiva que a de KK nas pacientes que não apresentavam cirurgia prévia para incontinência urinária.
A escolha de uma técnica cirúrgica não pode ser aleatória. Um dos fatores de confusão de nossa amostra foi que as indicações para o uso de uma técnica vaginal ou abdominal não foram as mesmas. A presença de prolapsos proeminentes das paredes vaginais ou mesmo do útero facilitou a indicação da via vaginal. Por isso, a análise das taxas de recidiva poderia ser diferenciada com os graus diferentes de prolapsos, o que na nossa amostra não foi possivel devido ao pequeno número de pacientes em cada subgrupo.

Embora não-significativa do ponto de vista estatístico (talvez em razão do pequeno número), encontramos uma alta prevalência de recidiva nos grupos abordados por via abdominal, principalmente no grupo submetido à cirurgia de MMK, no qual $75 \%$ das pacientes que recidivaram apresentavam história de cirurgia prévia. Ao excluirmos as pacientes com cirurgia prévia, a prevalência de recidiva mostrou diminuição mais importante nesses grupos. Assim, devemos considerar a possibilidade de encontrar, em uma amostra maior, influência significativa de cirurgia prévia na recidiva de incontinência urinária de esforço. É de salientar que cem por cento das pacientes com cirurgia prévia, não importando a técnica, apresentaram recidiva em dois anos de observação.

O climatério e suas conseqüências tem sido sistematicamente associados com piora do prognóstico dos prolapsos e da incontinência urinária. $\mathrm{Na}$ nossa amostra, a idade e a freqüência de pacientes já na menopausa em relação à recidiva não foram diferentes nos três tipos de cirurgia empregados. As pacientes submetidas à técnica de Marshall-Marchetti-Krantz tinham um número significativamente menor de anos de menopausa quando da realização da cirurgia quando comparadas com os outros dois grupos.

$O$ fato de em nossa amostra termos constatado um maior número de pacientes submetidas à perineoplastia posterior associada no grupo $1 \mathrm{lp}$ $=0,000015$ ) voltou a ser significativo nas pacientes desse mesmo grupo que não recidivaram quando comparadas com as que recidivaram. Isso anula a influência que o dado inicial poderia ter sobre as recidivas, pois, diferentemente dos dados encontrados na literatura, em nossa amostra as pacientes submetidas à cirurgia de Kelly-Kennedy com perineoplastia posterior associada recidivaram menos do que as pacientes que não receberam esta abordagem cirúrgica.

A escolha da técnica cirúrgica a ser empregada apresenta muito mais aspectos subjetivos do que objetivos. Existe uma tendência a verificarmos melhores resultados, em diversos autores, para as cirurgias abdominais, especialmente para a cirurgia de Burch. Na nossa amostra, não hou- 
ve diferença significativa nos três grupos estudados, principalmente quando retiramos o fator da realização de cirurgia prévia, que poderia intervir nos resultados. Observamos, entretanto, que a totalidade das pacientes com cirurgia prévia recidivou, nas três diferentes técnicas. Este dado nos conduz para melhor avaliar estas pacientes do ponto de vista diagnóstico e, até mesmo, de repensarmos a indicação cirúrgica quando da recidiva.

A pesquisa de modificadores de risco para incontinência urinária genuína não encontrou diferença significativa entre os três tipos de cirurgias estudados. A única característica mais freqüente foi a de anos de menopausa no grupo em que se realizou cirurgia de Burch, o que não influenciou a taxa de recidiva após dois anos de observação.

\section{SUMMARY}

Purpose: to analyze the prevalence of genuine urinary incontinence (GUI) recurrence, after at least two years of follow-up, in different surgical techniques used for its correction.

Patients and Methods: fifty-five patients with diagnosis of GUI, submitted to surgery for its repair at the Serviço de Ginecologia e Obstetrícia do Hospital de Clínicas de Porto Alegre from 1992 to 1996 and whose post-surgical followup was superior to 2 years were divided into three groups according to the surgical approach: Kelly-Kennedy $(n=$ $24)$, Burch $(n=23)$ and Marshall-Marchetti-Krantz $(n=8)$. Results: there were no differences regarding recurrence rate, age at surgery and at recurrence time, estrogen therapy, number of pregnancies and vaginal delivery $(p>0.05)$. Although posterior perineoplasty was more prevalent in the Kelly-Kennedy group, it did not influence the recurrence rate. The group submitted to the Burch approach had more years of menopause at the time of surgery.

Conclusion: the recurrence rates of urinary incontinence comparing the three different techniques (Kelly-Kennedy, Burch and Marshall-Marchetti-Krantz) were, respectively, 29.2, 39.1 and 50\%, which did not differ statistically. Considering the potential confusional bias for urinary stress incontinence, they did not differ among the groups. Nevertheless, we noticed that all women who had previous surgery presented recurrence of incontinence.

KEY WORDS: Stress urinary incontinence. Vaginal surgery. Hormonal replacement therapy.

\section{Referências}

1. Enzelsberger H, Helmer H, Schatten C. Comparison of Burch and lyodura sling procedures for repair of unsuccessful incontinence surgery. Obstet Gynecol 1996; 88: 251-6.

2. Ramos JG, Deos LR, Martins-Costa S, Lima C. Incontinência urinária. In: Freitas F, Menke CH, Rivoire W, Passos EP Rotinas em Ginecologia. $3^{\text {a }}$. ed. Porto Alegre: Artes Médicas; 1997. p. 12235.

3. Rodrigues Netto Júnior N. Incontinência urinária de esforço na mulher. Rev Assoc Med Bras 1990; 36: 15-7.

4. Girão MJBC, Sartori MGF, Kobata SA, Andrade FM, Juliano Y, Baracat EC, et al. Tratamento cirúrgico da incontinência urinária de esforço: análise de resultados pelas vias abdominal e vaginal. Rev Assoc Med Bras 1995; 41: 397-400.

5. Marana HRC, Andrade JM, Sala MM, Duarte G, Marana RRNF. Evaluation of long-term results of surgical correction of stress urinary incontinence. Gynecol Obstet Invest 1996; 41:214-9.

6. Colombo M, Milani R, Vitobello D, Maggioni A. A randomized comparison of Burch colposuspension and abdominal paravaginal defect repair for female stress urinary incontinence. Am J Obstet Gynecol 1996; 175: 78-84.

7. Beck RP, McCormick S, Nordstrom L. A 25-year experience with 519 anterior colporrhaphy procedures. Obstet Gynecol 1991; 78: 1011-8.

8. Harris RL, Yancey CA, Wiser WL, Morrison JC, Meeks GR. Comparison of anterior colporrhaphy and retropubic urethropexy for patients with genuine stress urinary incontinence. Am J Obstet Gynecol 1995; 173: 1671-5.

9. Kelly MJ, Knielsen K, Bruskewitz R, Roskamp D, Leach GE. Symptom analysis of patients undergoing modified Pereyra bladder neck suspension for stress urinary incontinence. Preand postoperative findings. Urology 1991; 37: 213-19.

10.Eriksen BC, Hagen B, Eik-Nes SH, Molne K, Mjolnerod OK, Romslo I. Long-term effectiveness of the Burch colposuspension in female urinary stress incontinence. Acta Obstet Gynecol Scand 1990; 69: 45-50.

11.Baden WF, Walker T, Kuehl TJ, Hillis A. The data base, data analysis, and biostatistics: a retrospective study of 864 patients. In: Baden WF, Walker T, editores. Surgical Repair of Vaginal Defects. Philadelphia: J.B. Lippincott; 1992. p. 77-96. 